\title{
Desarrollismo pedagógico en el programa de Tecnología Agropecuaria del Politécnico Colombiano Jaime Isaza Cadavid
}

\author{
Jorge A. Palacio-Martínez ${ }^{(1)}$, Francisco J. Hinojo-Lucena ${ }^{(2)}$ y Antonio M. Rodríguez-García(2) \\ (1) Facultad de Ciencias Agrarias, Politécnico Colombiano J.I.C., Cra 30 N 5F-185, Medellín-Colombia. \\ (e-mail: japalacio@elpoli.edu.co) \\ (2) Facultad de Ciencias de la Educación, Universidad de Granada, Avda. del Hospicio, s/n C.P. 18010, \\ Granada-España. (e-mail: fhinojo@ugr.es; arodrigu@ugr.es)
}

Recibido Nov. 20, 2018; Aceptado Ene. 25, 2019; Versión final Mar. 29, 2019, Publicado Oct. 2019

\begin{abstract}
Resumen
Este artículo presenta resultados de una investigación que analizó el currículo del programa de Tecnología Agropecuaria del Politécnico Colombiano Jaime Isaza Cadavid, abordando aspectos relacionados con la corriente desarrollista, el desarrollo rural y algunos factores internacionales que moldearon la educación técnica y tecnológica en Colombia. El enfoque metodológico fue el mixto, los datos cualitativos se obtuvieron a partir del análisis documental y entrevistas, los cuantitativos mediante la aplicación de un cuestionario a los actores del currículo. El análisis mostró que las normas aplicables a la educación tecnológica y el Sistema Nacional de Educación Terciaria, mantienen la idea original desarrollista. Basados en los resultados del estudio se concluye que el marco legal y el programa, privilegian el modelo curricular racional, con orientación al mercado y perspectivas teóricas funcionalistas, sin conexión con la dimensión socio-cultural prevista en el enfoque territorial rural.
\end{abstract}

\section{Pedagogical Developmentalism Agricultural Technology Program of the Politécnico Colombiano Jaime Isaza Cadavid}

\begin{abstract}
This article presents the results of a research that analyzes the academic curriculum of the Agricultural Technology program of the Politécnico Colombiano Jaime Isaza Cadavid, incorporating aspects related to the developmentalism current, the rural development, and some international factors that molded technical education and technology in Colombia. The methodological focus was mixed, with qualitative data obtained from a bibliographic review and interviews. Quantitative data was obtained through the use of a questionnaire administered to curricular actors. The analysis showed that the norms applicable to technological education and the National System of Higher Education maintain the original developmentalism idea. Based on the results of the study, it was concluded that both the legal framework and the program emphasize on the rational curricular model, and have market tendencies and functionalist theories without connections to the socio-cultural dimension seen in the rural territorial approach.
\end{abstract}

Keywords: developmentalism; education laws; technological education; curriculum model; territorial approach 


\section{INTRODUCCIÓN}

La expectativa de integrar a Colombia a la modernidad y al progreso técnico por la vía del desarrollo económico, en la segunda mitad del siglo XX, generaría nuevas oportunidades ocupacionales y demandas de personal calificado requeridos por el sector productivo, para las cuales el "modelo universitario tradicional, restringido, selectivo y elitista" no podía responder adecuadamente (Gómez, 2015). Adicionalmente, desde los años cuarenta, la política agraria de Colombia hizo énfasis en los instrumentos dirigidos a la modernización de la agricultura, la teoría prevaleciente sobre el desarrollo era la teoría de la modernización y esto suponía que el desarrollo y el progreso del país sólo se podía alcanzar a través de la "implementación de un sistema de educación superior orientado por la enseñanza de la técnica y la tecnología" (Acevedo, 2015). El proceso de expansión y diferenciación de la estructura económica, originada en la corriente desarrollista nacional, generó procesos de movilidad social que obligaron al Estado Colombiano a adaptar y diversificar el sistema de educación superior, expidiendo normas y creando nuevas tipologías educativas técnicas y tecnológicas, coherentes con el modelo de desarrollo, a la medida del mercado y de fácil masificación. En este marco surge en el año 1963 el Politécnico Colombiano Jaime Isaza Cadavid de Medellín-Colombia y en 1964 se crea el Programa de Tecnología agropecuaria.

La educación Técnica y tecnológica en el área agraria en Colombia, debe mirarse históricamente a partir de los cambios introducidos en las décadas del 50 y 60 del siglo pasado, debido a que son producto de factores internacionales que afectaron el diseño e implementación de políticas públicas y contribuyeron a moldear el marco legal, institucional y organizacional de estos niveles de educación superior. El entorno en el cual emergen como nuevas tipologías los Institutos Politécnicos y los programas técnicos y tecnológicos del sector agrario en Colombia, está estrechamente relacionado con las teorías del desarrollo de la modernización y la forma como estas teorías se expresaron, en las misiones económicas extranjeras, la Alianza para el Progreso y la revolución verde, en un contexto caracterizado por la división internacional del trabajo, los movimientos sociales y la transformación agraria centrada en la industrialización (Bernstein, 2016). De acuerdo con Wallerstein (2005) a partir de 1945 se estaba dando "un nuevo sentido de afirmación colectiva en América Latina", que coincide con el momento en que se "codifica el término desarrollo" y agrega este autor que, en un momento posterior a esta fecha, a esta "ideología", algunos autores latinoamericanos le darían el nombre de "desarrollismo". En ese contexto, la noción de desarrollo estaba basada en una teoría de estadios, de progreso lineal, dando por hecho que el país tecnológicamente más desarrollado, podía ser modelo para los países menos desarrollados (Wallerstein, 2005), lo cual según W. W. Rostow, permitiría el tránsito de una "sociedad tradicional a formas modernas de organización social" (Chamedes, 2015). Desde la década del cincuenta e incluso años antes, los países latinoamericanos adoptaron el desarrollismo nacional, como una estrategia basada en la economía del desarrollo y en la escuela estructuralista Cepalina (1953- 1955), proponiendo un modelo de Industrialización por Sustitución de Importaciones, de acuerdo con Pachón, Bokelmann y Ramírez (2016), el estructuralismo fue la forma en que la perspectiva de la modernización se transformó en políticas públicas en los países en desarrollo, con dos tendencias: "el modelo centro-periferia", que para el caso del sector agrario buscaba aumentar la producción mediante la industrialización de las zonas urbanas y "la teoría de la dependencia", que advertía que los países en desarrollo mantenían esa condición de dependencia debido a que no tenían acceso a la tecnología, en consecuencia, el desarrollo solo se podía lograr por la vía de la "industrialización y la aplicación de las nuevas tecnologías".

En relación con el sistema educativo, diversos estudios señalan que a partir de los años cincuenta y hasta mediados de los años ochenta del siglo pasado, las tendencias pedagógicas en América Latina y los modelos para el diseño curricular, tienen como marco general de referencia el desarrollismo, donde surgen "concepciones pedagógicas economicistas, tecnocráticas y modernizantes" (Galvani, 1990). El sistema educativo de algunos países latinoamericanos estaba sustentando en dos grandes tendencias: La primera hace referencia al desarrollismo pedagógico, encarnando la teoría de la dependencia y transformándose como lo plantea Galvani (1990) "en la última gran utopía educativa americana". Como narrativa dominante el desarrollismo pedagógico se consideró valido para todas las sociedades, sin tener en cuenta la identidad cultural del territorio y el escenario de construcciones sociales propias de cada país, imponiendo los valores y estilos de vida idealizados por occidente. La premisa del desarrollismo era el progreso de la sociedad y las habilidades socialmente útiles, razón por la cual era necesario crear nuevas tipologías educativas, que armonizaran con el modelo pedagógico modernizante de la educación superior, apelando entre otros mecanismos, a la formación de capital humano del nivel técnico y tecnológico. La segunda hace referencia a que el campo de la teoría curricular, también se vio permeado por la teoría de la modernización, en tal sentido, el sistema educativo acudió a un modelo de currículo eficaz, que permitiera formar mano de obra calificada para la toma decisiones racionales, eficaces y eficientes, es decir, un perfil profesional técnico, ello explica porque en el contexto del desarrollismo nacional, adquirieron relevancia los autores inscritos en el modelo de racionalidad tecnológica, tales como R. Tyler, H. Taba, M. Johnson y R. Mager (Díaz, 2003). Para el caso de los programas técnicos y tecnológicos del sector agrario, el modelo de la racionalidad 
tecnológica, estaba en sintonía con el enfoque de "desarrollo rural tecnocéntrico" (Pachón, Bokelmann y Ramírez, 2016), fundamentado entre otros aspectos, en el paquete tecnológico de la revolución verde, a través del cual se buscaba incrementar la productividad y maximizar los beneficios económicos, sin considerar las externalidades negativas de carácter social, ambiental e institucional derivadas de la aplicación de estas nuevas tecnologías.

Factores internacionales que contribuyeron a moldear la educación técnica y tecnológica del área agraria en Colombia

Un primer factor fue el papel que ejercieron las misiones económicas extranjeras y los organismos multilaterales creados después de la segunda guerra mundial, en la definición de políticas públicas, el establecimiento de cierto tipo de instituciones, la elaboración de diagnósticos y recomendaciones sobre el planeamiento educativo y la cuestión agraria en Colombia. Dos misiones para destacar: la misión Currie (1949 y 1953) abogando por un plan de industrialización con el propósito de "romper con los círculos viciosos del subdesarrollo y absorber la enorme masa de pobres rurales que permanecían en el campo en una situación de desempleo disfrazado" (Sandilands, 2018) y la misión Lebret (1958), proponiendo fortalecer la educación agrícola para impulsar el desarrollo. Sincrónicamente hay que resaltar, la influencia en el diseño e implementación de políticas de desarrollo, reforma agraria y educativa, que ejercieron organismos multilaterales como la Organización de Estados Americanos, el Banco Interamericano de Desarrollo y la Agencia Internacional de Desarrollo, creados respectivamente en los años 1948, 1959 y 1960. Soto y Forero (2016) plantean que, bajo el amparo de las directrices trazadas por las misiones norteamericanas y la CEPAL, en el contexto de los años sesenta, resultaba relevante que la universidad formara "mano de obra para el mercado", como una vía de acceso a la educación superior, para jóvenes provenientes de sectores sociales de menores recursos, coincidiendo este período con la crearon de los Institutos Politécnicos y las universidades tecnológicas de carácter regional. Al respecto Gómez (2015) manifiesta que "entre 1958 y 1964 se crearon varios institutos politécnicos postsecundarios en Colombia" de carácter público, entre los cuales menciona al Politécnico Jaime Isaza Cadavid de Medellín. Bajo esta coyuntura, los planes de desarrollo de Colombia también estuvieron direccionados por las misiones económicas extranjeras, Szegedy (2017) expresa que, "siguiendo las ideas de Lauchlin Currie", en el plan de desarrollo "las cuatro estrategias" (1970-1974), del presidente M. Pastrana se abogaba por la necesidad de aumentar la productividad agrícola y la tecnificación de los cultivos.

Un segundo factor internacional fue el programa de la Alianza para el Progreso propuesto en 1961 por John F. Kennedy, dirigido a frenar los fenómenos de exteriorización del descontento social y político que experimentaba Latinoamérica en los años sesenta y a generar condiciones favorables para el desarrollo, la estabilidad política y el bienestar de la población rural evitando la expansión de la revolución (LeGrand, et al., 2017; Dunne, 2016). La Alianza para el Progreso impulsó una "universidad modernizante", diferente a la universidad tradicional incapaz de responder satisfactoriamente a los programas de cambio propuestos por los Estados Unidos de Norteamérica (Marín, 2016). Justamente en el marco de la Alianza para el progreso, uno de sus emisarios, el asesor norteamericano Rudolf Atcon, presenta en 1961 un informe en el cual, de acuerdo con Acevedo (2015) este planteaba la necesidad de transformar la universidad, debido a que se encontraba aún "aferrada al pasado medieval", en menoscabo de la formación de profesionales que aplicaran "la ciencia y las técnicas modernas" al desarrollo social y económico de los países.

Un tercer aspecto no menos relevante, está relacionado con la influencia que ejerció y sigue ejerciendo la revolución verde, en el diseño curricular de los programas del área de las ciencias agrarias de educación superior. Según Raj (2013) el término revolución verde "fue acuñado por William Gaud al final de su desarrollo, en una reunión que se celebró en Washington DC en 1968". Se trató de un enfoque de desarrollo rural creado hacía principios de la década de 1940, pero con especial trascendencia en los años sesenta, liderado por el científico norteamericano Norman Borlaug, enmarcado en una visión tecnocentrista, de agricultura intensiva moderna, con incidencia principalmente en América Latina y Asia, secundado por compañas nacionales y respaldado por varios centros internacionales de investigación (Pachón, Bokelmann y Ramírez, 2016). La Revolución verde fue el resultado de los avances científicos del paquete tecnológico agrario, constituido por semillas de alto rendimiento, fertilizantes de síntesis, plaguicidas, maquinaria y combustible, los cuales produjeron aumentos considerables en el rendimiento agrícola de los principales cultivos comerciales del mundo, no obstante, en la mayoría de los casos, esos mayores rendimientos se obtuvieron a expensas del medio ambiente y del bienestar y la calidad de vida de las comunidades rurales (Gras y Hernández, 2016). En ese contexto, el currículo de los programas técnicos y tecnológicos se utilizó como un medio para instrumentalizar, difundir y extender, las técnicas modernizantes representadas por "el paquete tecnológico" propuesto por la revolución verde. La nueva tecnología rotulada por sus promotores de "moderna", estaba dirigida a la agricultura tradicional, conformada en su momento por agricultores y campesinos pobres, analfabetos o con bajos niveles de escolaridad. Quienes pudieron acceder a estas nuevas tecnologías, se hicieron dependientes de empresas multinacionales productoras de insumos 
procedentes de otros países, sobre los cuales los agricultores y técnicos no podían ejercer ninguna influencia. Esta ruptura en los patrones de cambio tecnológico del sector agrario, tendría y continúa teniendo importantes repercusiones económicas, ambientales, sociales, culturales e institucionales en el medio rural colombiano (Palacio, Hinojo y García, 2018).

En el marco del acuerdo de paz alcanzado con las fuerzas armadas revolucionarias de Colombia, ejército del pueblo (FARC-EP) en 2016, se acordó promover el desarrollo con enfoque territorial, con el fin de avanzar hacia una visión multidimensional e integral del desarrollo rural, a partir del reconocimiento de la diversidad de los territorios rurales, lo cual implica formar capital humano capaz de llevar a cabo procesos de intervención y de gestión diferenciados. Siguiendo a Posner (2004), un análisis del currículo del programa de tecnología agropecuaria llevado a cabo a partir de documentos oficiales y consulta de fuentes primarias, empleando algunos instrumentos aplicados a los diferentes actores del currículo, arrojó como resultado que, con algunas variantes obvias, actualmente existen renovadas narrativas desarrollistas en el marco legal, en los lineamientos de política pública y en el modelo de currículo del programa de tecnología agropecuaria, mostrando que este último no es pertinente con el enfoque territorial rural.

\section{METODOLOGÍA}

La unidad de análisis de la investigación fue el programa de Tecnología Agropecuaria del Politécnico Colombiano Jaime Isaza Cadavid de Medellín-Colombia. El trabajo se llevó a cabo entre el año 2012 y 2017. El enfoque metodológico fue el Mixto. En la fase cualitativa siguiendo a Posner (2004), se realizó un análisis documental del programa entre 1964 y 2016, que incluyó el marco normativo, las actuales políticas públicas y la identificación del modelo de currículo a partir de los documentos oficiales. Para complementar la información referida al modelo de currículo, se aplicó una entrevista semiestructurada a las dos directivas académicas más importantes del programa. En la fase cuantitativa se aplicó un cuestionario a los actores del currículo. La mayoría de ítems del cuestionario, hicieron énfasis en las cuatro (4) dimensiones del enfoque territorial rural: económica-productiva, sociocultural, ambiental y político-institucional. Los detalles de la metodología se presentan en la tabla 1.

Tabla 1: Metodología propuesta por Posner (2004) para el análisis del currículo y las fuentes documentales.

\begin{tabular}{|c|c|c|}
\hline \multicolumn{2}{|c|}{ Fase cualitativa } & Fase cuantitativa \\
\hline Análisis documental & Entrevistas & Cuestionarios \\
\hline $\begin{array}{l}\text { El análisis documental del } \\
\text { programa, se realizó siguiendo la } \\
\text { metodología para el análisis del } \\
\text { currículo propuesta por Posner } \\
\text { (2004), la cual consta de un } \\
\text { conjunto de } 68 \text { preguntas } \\
\text { organizadas en cuatro grupos. Los } \\
\text { resultados que al respecto se } \\
\text { presentan en este artículo } \\
\text { responden a algunas de las } \\
\text { preguntas del "grupo II". ¿Qué } \\
\text { situación produjo el desarrollo del } \\
\text { currículo?; 1) ¿A qué problema } \\
\text { social, económico, político o } \\
\text { educativo pretendía responder el } \\
\text { currículo?; 2) ¿Qué elementos de } \\
\text { planificación dominaron el proceso } \\
\text { de desarrollo del currículo? }\end{array}$ & $\begin{array}{l}\text { Preguntas: 1) ¿Cuál es la } \\
\text { metodología que se lleva a cabo en la } \\
\text { Facultad para el diseño o rediseño } \\
\text { curricular de los programas?; 2) ¿Hay } \\
\text { algunas etapas o fases que tengan } \\
\text { que cumplirse en el proceso de } \\
\text { diseño o rediseño curricular?; 3) } \\
\text { ¿Cómo se definen los propósitos del } \\
\text { programa? ¿Quiénes intervienen?; 4) } \\
\text { ¿Cómo se realiza el proceso de } \\
\text { selección de contenidos curriculares? } \\
\text { ¿Quiénes los seleccionan?; 5) } \\
\text { ¿Usted considera que el modelo } \\
\text { desarrollista que el Politécnico } \\
\text { declara en el modelo educativo, } \\
\text { incide realmente en la práctica } \\
\text { docente?; } 6 \text { ) ¿Cuáles son los criterios } \\
\text { que se tienen en cuenta para la } \\
\text { organización de currículo?; 7) ¿Cómo } \\
\text { se evalúa actualmente el currículo? }\end{array}$ & $\begin{array}{l}\text { Los datos cuantitativos, se obtuvieron } \\
\text { mediante la aplicación de cuatro (4) } \\
\text { cuestionarios estructurados, dirigidos } \\
\text { a los profesores, estudiantes, } \\
\text { egresados y empleadores, } \\
\text { autoaplicados, tipo likert de respuesta } \\
\text { graduada y de selección. Los } \\
\text { instrumentos se validaron mediante el } \\
\text { juicio de expertos, para tal efecto se } \\
\text { aplicó el coeficiente de Alfa de } \\
\text { Cronbach y para determinar la } \\
\text { consistencia entre jueces, se empleó } \\
\text { el coeficiente W. de Kendall. El } \\
\text { procesamiento de datos y análisis } \\
\text { estadístico, se realizó a través del } \\
\text { SPSS Statistics } 22\end{array}$ \\
\hline
\end{tabular}

\section{RESULTADOS Y DISCUSIÓN}

A continuación, se presentan los resultados y discusión de la fase cuantitativa y cualitativa.

Análisis documental. Marco legislativo aplicado a la educación tecnológica en Colombia

El análisis histórico del marco legal indica que el proceso de diversificación de las instituciones de educación superior en Colombia, se oficializa mediante el Decreto 1464 de 1963, "Por el cual se establecen normas 
para la fundación, aprobación e inspección de entidades no universitarias de Educación Superior". Este decreto establece que se entienden como institutos o escuelas de educación Superior los que, sin formar parte de una Universidad, mantienen estudios de formación de profesionales "mediante carreras cortas, intermedias o auxiliares que exigen el título de bachiller $u$ otro de igual nivel como requisito básico de ingreso". Luego en 1974 se expide el decreto 1358, este introduce la educación tecnológica como nueva tipología, señalando que, "los establecimientos de Educación Superior son institutos de educación tecnológica cuando sus programas tienen por objeto desarrollar destrezas y habilidades relacionadas con una tecnología específica, e impartir los conocimientos generales y científicos en que ésta se fundamenta". En relación con la educación tecnológica para el sector agrario, es preciso señalar que esta tipología se gesta en un entorno social y cultural con una incipiente institucionalidad educativa, de investigación, enseñanza y extensión de las ciencias agropecuarias, que poco pudieron aportar en ese momento histórico, a la construcción de una definición teórica y técnica empírica de lo que significa educar en tecnología para el sector agrario, este escenario pone en duda, la existencia de un marco conceptual auténtico para seleccionar las tecnologías relevantes y poder realizar adecuadamente la trasferencia de conocimientos científicos y tecnológicos a los programas de este nivel educativo. Basta con recordar que el Instituto Colombiano Agropecuario se crea en junio de 1962 y 1968 se crea El Consejo Nacional de Ciencia y Tecnología y el fondo Colombiano de Investigaciones Científicas y Proyectos Especiales "Francisco José de Caldas" (COLCIENCIAS).

En 1976 se expide el decreto 2667, "por la cual se definen y se reglamentan las carreras tecnológicas", allí se consagra que, "las carreras tecnológicas son un medio apropiado para formar personal técnico de nivel superior, requerido por la economía del país". El artículo 3oㅡ plantea que las carreras tecnológicas se definen porque tienen: "1) Orientación técnica, entendiéndose por tal su enfoque ocupacional de preparar para el ejercicio idóneo de una profesión, mediante el dominio de la tecnología correspondiente; 2) Plan de estudios con un $50 \%$ mínimo de instrucción práctica". El artículo $4^{\circ}$ expresa que, estos programas tienen como objetivos educativos "preparar personal técnico de alto nivel con miras a satisfacer los requerimientos de personal calificado planteados por los procesos de desarrollo socioeconómico del país". El artículo $7^{0}$ establece que el diseño curricular de los programas tecnológicos, "debe partir de un análisis ocupacional y de una descripción de los posibles campos de ejercicio profesional, determinantes del perfil educativo del egresado correspondiente". En el marco de este decreto se evidencia claramente la convergencia entre la narrativa de intervención económica, política e ideológica de los años sesenta y setenta, y la configuración de tipologías emergentes en la educación superior en Colombia. El Artículo 8o del mismo decreto manifiesta que, las asignaturas que conforman los planes de estudios para las carreras tecnológicas se "dosificarán y organizarán así: 1) 30\% de ciencias básicas (Matemáticas, Física, Química, Biología) ; 2) 50\% de tecnología específica; 3) 10\% de tecnologías complementarias; 4) 5\% de técnicas de comunicación; 5) 5\% de cultura general". La duración de los estudios será de seis (6) semestres o su equivalente en períodos académicos. Aquí se percibe claramente cómo se minimiza la formación humanística en el diseño de estos programas. En relación con el artículo $7^{\circ}$ antes mencionado, hay que anotar que, por primera vez en las normas aplicables a la educación tecnológica en Colombia, se mencionaba el término currículo. Al respecto, la mayoría de los historiadores, coinciden en que el uso del término como área de teorización e investigación, proviene de las ideas fundadoras del campo curricular publicadas por Franklin Bobbit en 1918; 1924, en los libros The Curriculum y How to make a curriculum, respectivamente. Según Posner (2004), Bobbit, establece un método básico de planificación curricular, seguido posteriormente por Tyler, R. y agrega que, desde la publicación del libro de Tyler en 1949, Basic principles of curriculum and instruction, "educadores que representan una amplia variedad de orientaciones han acudido al modelo Tyler para responder a las preguntas de procedimiento curricular", estas son: "1) ¿Cuáles propósitos educativos debe tratar de alcanzar la escuela?; 2) ¿Qué experiencias educativas deben ofrecerse para alcanzar estos propósitos?; 3) ¿Cómo se organizan estas experiencias de manera eficaz?; 4) ¿Cómo se determina si se han alcanzado estos objetivos?" de acuerdo con este mismo autor se trata de un currículo orientado a la producción, concentrado en sus productos. En esta misma línea, en 1962 Taba, H., publica el libro Curriculum development; theory and practice, las ideas expresadas por esta autora tuvieron una gran influencia especialmente en los años sesenta y setenta, y todavía son mencionadas con frecuencia por algunos autores del siglo XXI (Laanemets y Kalamees, 2013).

En 1980 se promulga el decreto 80, el artículo 25 establece cuatro "modalidades educativas" para la educación superior en Colombia: "1) Formación intermedia profesional; 2) Formación tecnológica; 3) Formación universitaria y 4) Formación avanzada o de postgrado". En lo que respecta a las modalidades de pregrado, el artículo $25^{\circ}$ ubica la formación tecnológica en el centro de una pirámide, abajo el nivel técnico y encima la formación universitaria, agudizando la tensión que existe entre la formación universitaria y el saber hacer ligado a la práctica y ampliando las disparidades en capacidades sociales, científicas y tecnológicas entre estas dos modalidades. En 1992 se expide la Ley 30 o Ley de Educación Superior para Colombia, aún vigente. La Ley no hace claridad sobre los alcances de la educación tecnológica, se limita a señalar que la tecnología es "un campo de acción de la educación superior", relacionado con el "desempeño 
de ocupaciones, para el ejercicio de una profesión o disciplina determinada, de naturaleza tecnológica o científica o en el área de las humanidades, las artes y la filosofía". Por esta época Colombia ya había iniciado un proceso de apertura económica, con la que buscaba integrarse a la economía global, pasando del proteccionismo económico implícito en el modelo de industrialización por sustitución de importaciones, a un sistema abierto a los demás países del mundo, lo cual implicaba tomar medidas para ajustar el gasto público y reducir el tamaño del Estado (Uribe,2014), esta coyuntura trajo consigo el desmantelamiento sistemático de la Institucionalidad del sector agrario en Colombia.

En 2002 se promulga la Ley 749, mediante la cual empieza a circular el concepto de formación por ciclos propedéuticos, a través de los cuales se pretendía articular los tres niveles de pregrado, técnico profesional, tecnológico y profesional. Esta Ley restringía la oferta de programas a "las áreas de las ingenierías, la tecnología de la información y la administración", hasta cuando se expide la Ley 1188 de 2008, en adelante, los ciclos propedéuticos se pudieron ofrecer "en todos los campos y áreas del conocimiento", incluyendo programas de tecnologías en desarrollo humano. Y entonces ¿De qué educación tecnológica se habla en Colombia?

\section{La educación terciaria en Colombia. Cambio sin innovación}

Actualmente en Colombia la educación superior se imparte en dos niveles: pregrado y posgrado. El nivel de pregrado incluye tres niveles de formación: el Técnico Profesional, el Tecnológico y el Profesional. En el año 2015, el Plan Nacional de Desarrollo 2014-2018, "Todos por un nuevo País", crea el Sistema Nacional de Educación Terciaria (SNET) (Ley 1753, 2015), el cual reorganiza la oferta de formación post-media en dos rutas o pilares: educación universitaria y educación profesional (técnica). El Pilar de Educación Técnica comprende los niveles de técnico superior, especialización técnica y especialización tecnológica. Los programas pertenecientes a este pilar, al cual pertenecería la educación tecnológica, tienen una clara orientación hacia el mercado laboral, como quiera que el diseño curricular se basaría en mapas funcionales sectoriales, normas de competencia laboral y estándares ocupaciones, tal y como se viene haciendo con los programas de Educación para el Trabajo y el Desarrollo Humano (antes llamada en Colombia educación no formal), es decir, un currículo prescriptivo, técnico-funcional, vocacional, que forme a la medida de ciertos sectores productivos, lo cual pone de manifiesto la concurrencia en la política pública de renovadas corrientes desarrollistas, ya que para el caso de los programas del área agraria, mantienen la idea original de convertir la agricultura tradicional en agricultura dirigida al mercado internacional. Weis y Bernal (2013) expresan que hacia la mitad de los años noventa, ocurrió algo similar con la educación tecnológica en México, proponiendo al igual que en Colombia, la adopción de un "modelo de educación basada en competencias, mediante la aplicación de las normas técnicas de competencia laboral como referentes del desarrollo curricular". Volviendo al Sistema Nacional de Educación Terciaria, lo que se observa es que se trata de una propuesta dicotómica, educación universitaria vs educación profesional (técnica), además regresiva ya que revive a través de la ruta profesional (Técnica), tipologías educativas validadas empíricamente de bajo reconocimiento social. De acuerdo con el Ministerio de Educación Nacional con la creación del sistema se corrigen problemas estructurales tales como, la desarticulación entre niveles educativos, ausencia de orientación vocacional, falta de pertinencia, desarticulación con el sector productivo, ausencia de un sistema integral de aseguramiento de la calidad y el bajo reconocimiento histórico de la calidad de los programas tecnológicos en Colombia.

Un antecedente que ilustra, lo que finalmente se aprobó como modelo de educación terciaria para el pilar de la educación Profesional (técnica), se encuentra en un documento oficial publicado en 2008 por el Ministerio de Educación Nacional, titulado "Educación Técnica y Tecnológica para la competitividad" (Ministerio de Educación Nacional, 2008). Este documento traza un conjunto de orientaciones, "para el diseño o transformación de programas pertinentes y de calidad", que de acuerdo con sus promotores, posibilitaría ampliar la demanda y "contrarrestar el imaginario negativo" existente alrededor de la educación técnica y tecnológica, "producto del gran desconocimiento que tiene el país sobre sus características, ventajas, pertinencia para el desarrollo y efectividad en términos de retorno de la inversión para las personas y sus familias". Allí se expresa que, un tecnólogo "desarrolla competencias relacionadas con la aplicación y práctica de conocimientos en un conjunto de actividades laborales", haciendo explicito el interés del Ministerio de Educación Nacional, por definir un perfil de tecnólogo, en el marco de la Clasificación Nacional de Ocupaciones, en un área de desempeño determinado y un nivel de cualificación para un ambiente laboral. En este documento se propone que los programas se diseñen y evalúen por competencias, de modo que sean comparables y compatibles con otros programas del mismo nivel o con pruebas estandarizadas como el Examen de Estado de Calidad de la Educación Superior, ICFES Saber-Pro. Los tipos de competencias para el diseño curricular, son las definidas en el marco del proyecto Tunnig: competencias básicas, genéricas o transversales, sistémicas y específicas, direccionadas principalmente desde el Espacio Europeo de Educación Superior, para ello, estos programas se deberían articular mediante alianzas, con los sectores productivos estratégicos, lo cual exigiría preparar a las instituciones, 
realizando "cambios sustanciales en la gestión académico-administrativa y en los modelos pedagógico y los métodos formativos" y "tomar decisiones de carácter político, académico y administrativo" (Ministerio de Educación Nacional, 2008). Basados en estos argumentos, el Ministerio de Educación considera que, esta sería la ruta para romper con las "ideas preestablecidas", que relacionan el objetivo de educación tecnológica, con la generación de "mano de obra barata" y que formar personas a la medida de las necesidades del sector productivo "es aceptar el capitalismo salvaje".

\section{Modelo de currículo del programa de tecnología agropecuaria a partir del análisis documental}

El Politécnico Colombiano Jaime Isaza Cadavid, tiene un procedimiento estándar para crear, actualizar, extender, ofrecer un programa o renovar el registro calificado o licencia de funcionamiento del programa, en el contexto de la educación superior en Colombia. Siguiendo a Posner (2004), un análisis de las "etapas" que incluye este procedimiento y el análisis detallado del currículo del programa de Tecnología Agropecuaria, entre 2004 y 2016, permitió evidenciar los supuestos de los cuales parte el programa:

1) Modelo curricular lineal de planificación por objetivos y/o racional claramente influenciados la teoría curricular de Tyler y Taba; 2) Práctica docente en la cual priman las perspectivas teóricas conductistas 0 funcionalistas; 3) Áreas del programa abordadas desde un enfoque saber disciplinar-técnico-sectorial y no a partir de campos del saber interrelacionados. En algunos casos, la selección de propósitos y contenidos de las asignaturas por parte de los profesores, no establece una relación explícita con el perfil de egreso. Al respecto Icarte y Labate (2016) encuentran situaciones similares en otros programas de educación superior; 4) escasa participación de los actores del currículo especialmente egresados, en el análisis del currículo; 5) Primacía en la selección de contenidos con enfoque analítico, en cambio, el área socio-humanístico se minimiza y se margina la práctica social rural; 6) Selección de contenidos que convergen en sus medios y fines, con la formación de tecnólogos para el mercado laboral. Los contenidos, se priorizan sobre el método y son asumidos como un conjunto de conocimientos, habilidades y destrezas, para desempeños eficaces en el contexto de sistemas de producción agropecuaria convencional, cadenas productivas y sectores de clase mundial; 7) Secuencia en la estructura de las disciplinas, hipotético-deductivo; 8) Ambientes de aprendizaje ubicados en la ciudad capital o en el área metropolitana; 9) Enfoque investigativo enmarcado en el paradigma cuantitativo; 10) Evaluación por productos o resultados, con enfoque cuantitativo. Con base en este análisis y como un criterio de responsabilidad social, la formación de los tecnólogos agropecuarios, no se puede continuar reduciendo a la formación de capital humano para el incremento de la competitividad de ciertos sectores económicos, menos aún en un país como Colombia, donde el medio rural ha sido objeto de un conflicto armado interno por más de cinco décadas y lo que se esperaría del proceso formativo, sería una contribución al logro de una sociedad rural, no solo productiva y competitiva, sino también equitativa, sostenible y solidaria (Palacio, Hinojo y García, 2018). De acuerdo con lo anterior, se hace necesario introducir transformaciones relevantes al currículo para pasar de un enfoque funcionalista orientado a la producción convencional, a un proceso de formación integral que incluya aspectos tales como: 1) Dinámica de los mercados locales, regionales, nacionales e internacionales; 2) Relaciones ciencia, tecnología e innovación para el desarrollo agropecuario y rural; 3) Transformación productiva, generación de valor agregado y competitividad; 4) Seguridad y soberanía alimentaria; 5) Articulación entre los sistemas naturales y los sistemas productivos y sociales; 6) Participación comunitaria en la planeación del territorio; 7) Investigación, validación e intercambio de tecnología para el medio rural; 8) Enfoque de desarrollo rural en Colombia; 9) Planes de Gobierno Nacional, Departamental y Municipal; 10) Asociatividad e inclusión económica y social de las comunidades rurales.

\section{Modelo de currículo a partir de las entrevistas a las directivas académicas}

El primer informante expresa que la metodología que se emplea para el diseño o rediseño del programa, se inicia a partir de un análisis del contexto, teniendo como referentes el Proyecto Educativo Institucional, el marco normativo y los lineamientos de carácter legal definidos por el Ministerio de Educación Nacional. Destaca el papel que cumple el Comité de Área y de Currículo. Valora las lecturas de realidades que se pueden hacer a partir de los encuentros con estudiantes, docentes de cátedra, egresados y el sector productivo. Menciona que, liderados por los docentes, es al interior de los Comités de Área y de Currículo, donde se analiza el currículo con fines de actualización y se definen las estrategias evaluativas y metodológicas de las diferentes asignaturas. En relación con las etapas para el diseño o rediseño curricular, enfatiza en la estructura organizacional, definiendo tres instancias jerárquicas así: el Comité de Área, de Currículo y el Consejo de Facultad. Es el Comité de Currículo quien debe convocar a los demás actores para definir los propósitos del programa, teniendo como referentes las áreas o componentes legalmente establecidas por el Ministerio de Educación Nacional, a partir de las cuales se deriva el plan de estudio y se definen los contenidos de las asignaturas. En cuanto al modelo pedagógico, manifiesta que, una cosa es lo que dice el Proyecto Educativo Institucional y otra lo que ocurre en la práctica, al respecto considera que existe un enorme vacío, dejando en manos de cada docente, aplicar su propio modelo de acuerdo con su 
conocimiento y experiencia. Para la organización del currículo propone la siguiente secuencia: misión, visión y objetivos del programa, definidos a partir de la realidad externa y en sintonía con la misión y visión institucional, luego propone definir el perfil asociado a competencias o capacidades.

El segundo informante manifiesta que la metodología que se emplea para llevar a cabo un proceso de diseño o rediseño curricular, se apoya en los cuerpos colegiados con que cuenta la institución, principalmente en los Comités de Currículo y de Área, teniendo como referente la metodología que establece el Ministerio de Educación Nacional y direccionado por la oficina de autoevaluación. En relación con las etapas para el diseño o rediseño curricular, considera que se parte originalmente de un análisis de pertinencia del contexto interno y externo, del tipo de profesional que se quiere formar y de las necesidades de la sociedad. Dentro de estas etapas también incluye el mercado internacional y la convergencia del programa con otros afines. En cuanto a la definición de los propósitos y contenidos, manifiesta que básicamente descansa en el Comité de Currículo y de Área. Los docentes asumen la responsabilidad de actualizar los propósitos y contenidos mediante formatos estándar diseñados institucionalmente para tal efecto. Frente a la incidencia del modelo pedagógico desarrollista declarado oficialmente en la Institución, considera que tanto los objetivos, como los procedimientos, han sido estandarizados en función de este modelo, sin embargo, opina que los docentes aún continúan inmersos en un modelo tradicional, no hay un modelo crítico, adicionalmente los ambientes de aprendizaje no convergen con este modelo. La organización del programa, especialmente el componente práctico, se ve afectado por los índices de repitencia de los estudiantes, lo cual dificulta los desplazamientos al medio rural. Finalmente centra la evaluación del currículo, en la opinión que al respecto emiten los docentes vinculados, de cátedra, estudiantes y personal administrativo, haciendo énfasis más en un concepto de autoevaluación, que de evaluación curricular (Palacio, Hinojo y García, 2018). Aquí es importante anotar que el Comité de currículo del programa, la mayoría de las veces, por no decir que siempre, sesiona sin presencia de los representantes estudiantiles, graduados y sector empleador, lo cual significa que todas las actividades relacionadas con la actualización curricular recaen en manos de los representantes profesorales y las directivas académicas.

\section{Algunos resultados de la fase cuantitativa}

La fiabilidad y consistencia interna de los cuestionarios se determinó por medio del método de consistencia interna, a través del coeficiente alfa de Cronbach y la consistencia entre jueces mediante la prueba W de Kendall. Los cuestionarios se aplicaron a la totalidad de los 40 profesores del programa, 77 estudiantes del quinto y sexto nivel, a 50 egresados con experiencia laboral y 19 empleadores representativos del sector, para un total de 186 individuos. Para todos los casos la prueba alfa de Cronbach estuvo por encima de 0.846 y de 0.218 para la prueba Kendall, estos índices aseguran una apropiada consistencia interna y concordancia significativa entre los rangos asignados por los jueces.

Los resultados de los procesos de autoevaluación realizados en 2006, 2010 y 2012, mostraron que, de todas las características evaluadas en los tres procesos, la más baja calificación la obtiene el impacto de los egresados en el medio social y académico. De otro lado, los resultados del análisis del currículo indican que el programa de tecnología agropecuaria minimiza y aísla el área socio-humanística de las otras áreas, en tal sentido, el componente humanístico del programa está conformado por asignaturas como humanidades I, pedagogía constitucional, deporte arte y recreación, y desarrollo rural, que equivalen al $8.3 \%$ de los créditos del programa, suficientes para enmascarar el concepto de formación integral declarado en el perfil, pero insuficientes para conectar al estudiante con la realidad del medio rural y sus protagonistas. Un análisis de frecuencia estadística con la que los profesores emplean algunos verbos, para definir los objetivos generales de las asignaturas elaboradas en diferentes momentos, 2004, 2007, 2008, 2009, mostró que el verbo que más se repite es conocer, distante de verbos como transformar o aplicar, por mencionar solo algunos. Cuando a los 77 estudiantes de los últimos dos niveles y a los 50 egresados del programa se les preguntó por la formación que recibieron en temas relacionados con el enfoque territorial, algunos de los resultados obtenidos se presentan en las figuras 1 y 2.

En el marco de los acuerdos logrados con las FARC-EP, el enfoque territorial rural se entiende como un proceso de transformación económica, social, ambiental e institucional de los territorios, en el cual los actores sociales locales tienen un papel preponderante. Con base en esta visión y como un principio de responsabilidad social universitaria, las instituciones de educación superior del área agraria, deberían contribuir a generar capacidades locales, para que las propias comunidades puedan gestionar su desarrollo, y a su vez, superen las intervenciones sectoriales, por un enfoque de desarrollo multidimensional del territorio (Esparcia et al., 2015). 


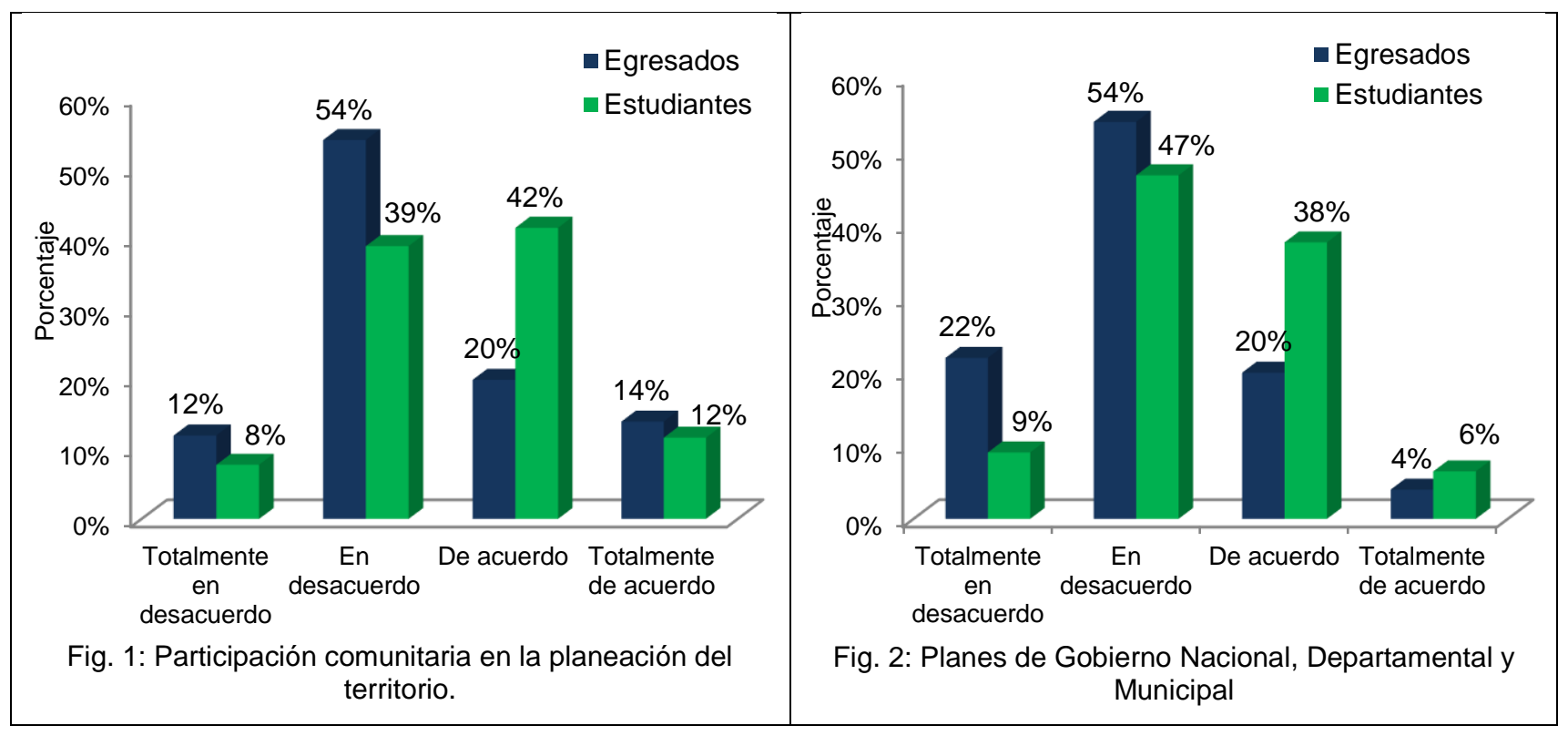

Pese a este interés nacional, en los lineamientos normativos y de política pública, así como en el propio diseño del programa de tecnología agropecuaria, la dimensión socio-cultural, ambiental e institucional se sigue planteando desligada de la dimensión productiva-sectorial. En relación con este último tema, un estudio llevado a cabo por Cáceres (2015) en argentina, mostró que la tecnología agropecuaria y los agronegocios, siguen estando dominados por "la lógica subyacente del modelo tecnológico dominante", representado por la "agricultura industrial". Agrega este autor que, en disciplinas como la agronomía, prima el área productivista sobre las áreas social y ambiental, y en ese sentido, hay una aplicación reduccionista de la tecnología, por cuanto no permite encarar desde un enfoque sistémico, "la complejidad y multidimensionalidad de los problemas agropecuarios" y rurales. Coincidiendo con estos hallazgos, las figuras 1 y 2 , muestran que, por ejemplo, los temas relacionados con la participación comunitaria en la planeación del territorio y los planes de gobierno, son marginales en el proceso formativo del tecnólogo agropecuario, a lo cual se le suma la falta de participación de los egresados en el diseño, desarrollo y evaluación del currículo. Figura 3.

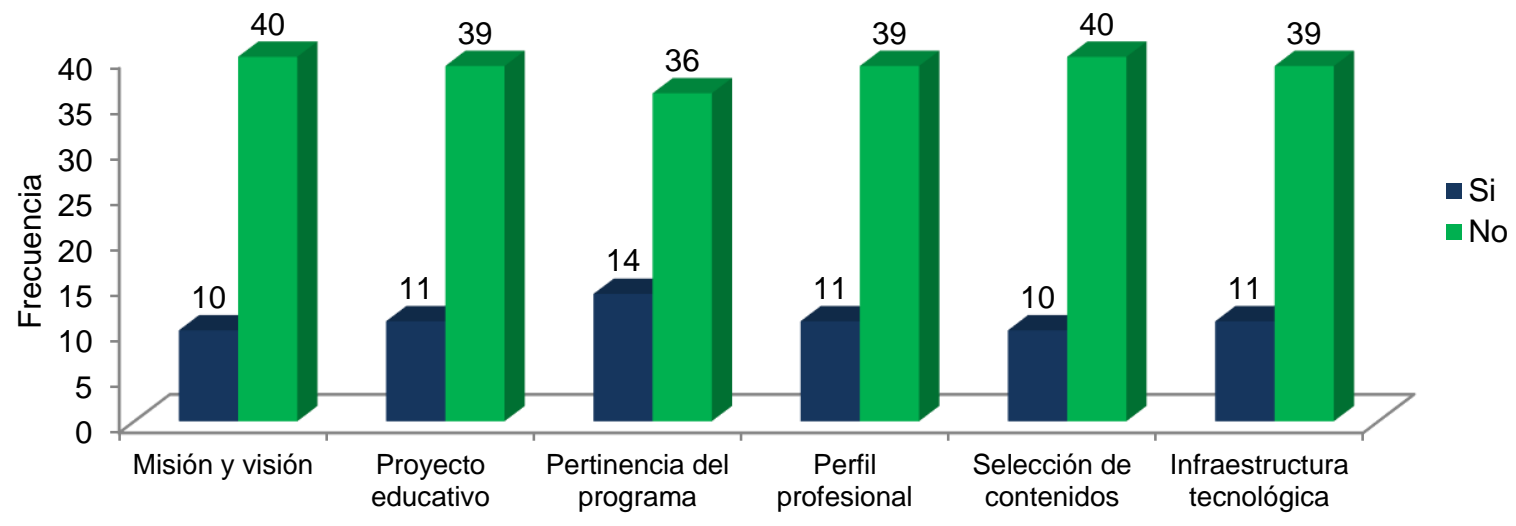

Fig. 3: Participación individual o colectiva en el análisis del currículo con aportes o sugerencias al programa

La figura 3 muestra que, a los egresados del programa, no se les brinda la oportunidad de participar activamente en la actualización del currículo, revisando su misión y visión, definiendo criterios de pertinencia en relación con las nuevas dinámicas en el área agropecuaria y rural, replanteando el perfil profesional y ocupacional, y lo que esto implica en términos de la selección de contenidos, metodologías e infraestructura tecnológica necesaria para llevar a cabo un proceso formativo de calidad. El egresado es un actor del currículo muy importante, que, de ser valorado, podría hacer importantes contribuciones en el direccionamiento colectivo del programa, haciéndolo más pertinente, entre otros aspectos, con el enfoque territorial rural. 


\section{CONCLUSIONES}

De los resultados mostrados, de su análisis y discusión, se pueden obtener las siguientes conclusiones: 1) El análisis histórico del marco legal y los lineamientos de política pública, evidencian la falta de innovación y el poder que sigue ejerciendo el contexto, entiéndase el mercado, en el diseño curricular los programas de educación tecnológica, incluyendo la tecnología agropecuaria. El pilar de educación técnica propuesto en el Sistema Nacional de Educación Terciaria en Colombia, sigue la lógica del desarrollismo pedagógico, ya que continúa privilegiando la visión productiva sectorial desligada de las otras dimensiones del desarrollo: sociocultural, ambiental e institucional; 2) las perspectivas teóricas y el modelo de currículo que privilegia el programa, indican que actualmente la tecnología agropecuaria no es pertinente con el enfoque territorial rural; 3) La escasa participación de los egresados en el análisis del currículo y la marginación de la práctica social situada en la ruralidad, explica porque en los tres de los procesos de autoevaluación analizados, la característica con la más baja calificación fue el impacto de los egresados en el medio social y académico; 4) Los problemas que afectan la educación tecnológica en Colombia tienen un origen multicausal relacionado entre aspectos, con las contradicciones normativas y la falta de claridad conceptual acerca de lo que significa formar en tecnología agropecuaria desde un enfoque multidimensional del desarrollo, esta situación ha contribuido a crear un imaginario colectivo que relaciona este tipo de educación con carreras para el desempeño de oficios prácticos, pobremente valoradas por la sociedad y el mercado de trabajo.

\section{REFERENCIAS}

Acevedo, A., Educación, reformas y movimientos universitarios en Colombia: apuestas y frustraciones por un proyecto modernizador en el siglo XX, doi: 10.7440/res53.2015.08, Revista de Estudios Sociales (53), 102-111 (2015)

Bernstein, H., Agrarian political economy and modern world capitalism: the contributions of food regime analysis, doi: 10.1080/03066150.2015.1101456, The Journal of Peasant Studies, 43(3), 611-647 (2016)

Cáceres, D., Tecnología agropecuaria y agronegocios. La lógica subyacente del modelo tecnológico dominante, Mundo Agrario, ISSN: 1515-5994, 16(31), 1-30 (2015)

Chamedes, G., The catholic origins of economic development after World War II, doi: 10.3167/fpcs.2015.330204, French Politics, Culture \& Society, 33(2), 55-75 (2015)

Decreto 1358 de 1974, Por el cual se dictan normas sobre educación superior, 1-2, Bogotá, Colombia (1974)

Decreto 1464 de 1963, Por el cual se establecen normas para la fundación, aprobación e inspección de entidades no universitarias de Educación Superior, 1-4, Bogotá, Colombia (1963)

Decreto 2667 de 1976, Por el cual se definen y reglamentan las carreras tecnológicas, 1-4, Bogotá, Colombia (1976)

Decreto 80 de 1980, Por el cual se organiza el sistema de educación postsecundaria, Bogotá, Colombia (1980)

Díaz, F., Main Trends of Curriculum Research In Mexico, 457-469. Taylor \& Francis e-Library, New Jersey, EEUU (2003)

Dunne, M., Review article Kennedy Alliance for Progress: countering revolution in Latin America Part II: the historiographical record, doi: 10.1111/1468-2346.12558, International Affairs, 92(2), 435-452 (2016)

Esparcia, J., J. Escribano y J. Serrano, From development to power relations and territorial governance: Increasing the leadership role of LEADER Local Action Groups in Spain, doi: 10.1016/j.jrurstud.2015.09.005, Journal of Rural Studies, 42, 29-42 (2015)

Galvani, V., Mirando al 92, el fin de una utopía educativa: el desarrollismo en América Latina. Revista complutense de Educación, ISSN: 1988-2793, 1(13), 477-485 (1990)

Gómez, V., La pirámide de la desigualdad en la educación Superior en Colombia. Diversificación y tipología de instituciones, $1^{\text {a }}$ Ed., 19-227, Universidad Nacional de Colombia, Bogotá, Colombia (2015)

Gras, C. y V. Hernández, Modelos de desarrollo e innovación tecnológica: una revolución conservadora, Mundo Agrario, ISSN: 1515-5994, 17(36), 1-27 (2016)

Icarte, G. y H. Labate, Metodología para la Revisión y Actualización de un Diseño Curricular de una Carrera Universitaria Incorporando Conceptos de Aprendizaje Basado en Competencias, doi: 10.4067/S071850062016000200002, Formación Universitaria, 9(2), 3-16 (2016)

Laanemets, U. y K. Kalamees, The Taba-Tyler Rationales. Journal of the American Association for the Advancement of Curriculum Studies, 1-12 (2013)

LeGrand, C., L. Isschot y P. Riaño, Land, justice, and memory: challenges for peace in Colombia, doi: 10.1080/08263663.2017.1378381, Canadian Journal of Latin American and Caribbean Studies, 42(3), 259-276 (2017)

Ley 1753 de 2015, Por la cual se expide el Plan Nacional de Desarrollo 2014-2018 Todos por un nuevo país, 1-104, Bogotá, Colombia (2015)

Ley 30 de 1992, Por la cual se organiza el servicio público de la Educación Superior, 1-26, Bogotá, Colombia (1992)

Ley 749 de 2002, Por la cual se organiza el servicio público de la educación superior en las modalidades de formación 
técnica profesional y tecnológica, 1-5, Bogotá, Colombia (2002)

Marín, M., Trayectorias, misiones e identidades de la Universidad latinoamericana, doi: 10.11600/1692715x.14211030815, Revista Latinoamericana de Ciencias Sociales, Niñez y Juventud, 14(2), 1041-1053 (2016)

Ministerio de Educación Nacional, Educación Técnica y Tecnológica para la competitividad, $1^{\text {a }}$ Ed., 11-84, Ministerio de Educación Nacional, Bogotá, Colombia (2008)

Pachón, F., W. Bokelmann y C. Ramírez, Rural development thinking, moving from the green revolution to food sovereignty, doi: 10.15446/agron.colomb.v34n2.56639, Agronomía Colombiana, 34(2), 267-276 (2016)

Palacio, J., M., Hinojo y S. García, Educación superior en Tecnología Agropecuaria con enfoque territorial, doi: 10.24310/innoeduca. 2018.v4i1.2453, Innoeduca. international journal of technology and educational innovation, 4(2), 159-171 (2018)

Posner, G., Análisis del currículo, $2^{\mathrm{a}}$ Ed., 1-372, McGraw-Hill, Ciudad de México, México (2004)

Raj, P., The Long Green Revolution, doi: 10.1080/03066150.2012.719224, The Journal of Peasant Studies, 40(1), 1-63 (2013)

Sandilands, R., Albert Hirschman, Lauchlin Currie, la teoría de los "eslabonamientos" y el "gran impulso" de Paul Rosenstein-Rodan, doi: 10.18601/01245996, Revista de Economía Institucional, 20(39), 53-68 (2018)

Soto, D. y A. Forero, La Universidad Latinoamericana y del Caribe en los desafíos del Siglo XXI, doi: 10.19053/01227238.4375, Revista Historia de la Educación Latinoamericana, 18(26), 279-309 (2016)

Szegedy, I., Agricultural Public Policies in Colombia from the 1970s through the Uribe Government - The Story of Recurrent Unresolved Policy Issues, doi: 10.11144/Javeriana.vj134.appc, Vniversitas, (134), 363-398 (2017)

Uribe, C., The Positioning of Economic Rationality in Colombia, Bulletin of Latin American Research, 33(1), 46-59 (2014) Wallerstein, I., After Developmentalism and Globalization, What? Social Forces, 85(3), 1263-1278 (2005)

Weiss, E. y E. Bernal, Un diálogo con la historia de la educación técnica mexicana, Perfiles Educativos, ISSN: $0185-$ 2698, 35(139), 151-170 (2013) 
